\title{
THOMAS-FERMI AND POISSON MODELING OF GATE ELECTROSTATICS IN GRAPHENE NANORIBBON
}

\author{
T. Andrijauskas ${ }^{\text {a }}$, A.A. Shylau ${ }^{\text {b }}$, and I.V. Zozoulenko ${ }^{\text {b }}$ \\ ${ }^{a}$ Institute of Theoretical Physics and Astronomy, Vilnius University, A. Goštauto 12, LT-01108 Vilnius, \\ Lithuania \\ E-mail: tomas.andrijauskas@tfai.vu.lt \\ b Solid State Electronics, Department of Science and Technology (ITN), \\ Linkoping University, 60174 Norrkoping, Sweden
}

Received 30 September 2011; revised 13 December 2011; accepted 1 March 2012

\begin{abstract}
We describe a simple graphene nanoribbon and bottom gate system and present numerical algorithms for solving Poisson's and Thomas-Fermi equations for electrons in the graphene nanoribbon. The Poisson's equation is solved using finite difference and finite element methods. Using the Poisson and Thomas-Fermi equations we calculate an electrostatic potential and surface electron density in the graphene nanoribbon. Finally, the Poisson-Thomas-Fermi model for the graphene nanoribbon is compared to a tight-binding Hartree model. The results show a good correspondence with the tight-binding model. The developed solver of the Poisson's equation can be used in the future calculations of more complex graphene and gate systems.
\end{abstract}

Keywords: graphene, Thomas-Fermi approximation, Poisson's equation

PACS: 81.05.ue, $78.35 .+\mathrm{c}$

\section{Introduction}

One of the most important discoveries in the condensed matter physics in the past decades is the experimental fabrication of graphene - a single sheet of carbon atoms arranged in a honeycomb lattice (Fig. 1). Since its discovery in 2004 [1], graphene has attracted an enormous attention because of its highly unusual electronic and transport properties that are strikingly different from those of conventional semiconductor-based two-dimensional electronic systems [2]. Graphene is a semimetal with no gap and zero density of states at the Fermi energy. Its unusual properties are due to the linear dispersion with particle-symmetry implying the constant speed of electrons independent on momentum. Its Hamiltonian is similar to that of two-dimensional massless Dirac fermions, which leads, for example, to such remarkable properties as Klein tunnelling, i. e. the reflectionless penetration of electrons through high and wide potential barriers.

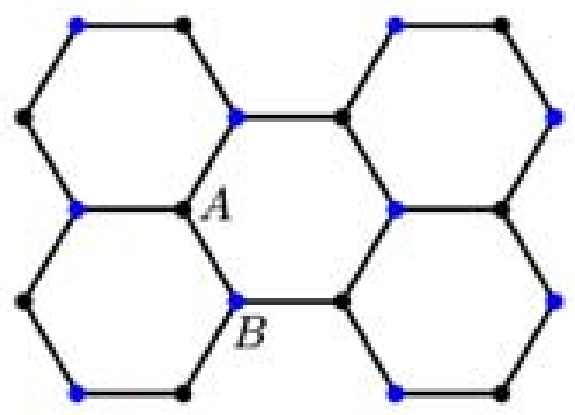

Fig. 1. Graphene lattice. The letters $A$ and $B$ indicate two different triangle sub-lattices.

While most of earlier studies have been focused on the basic properties of graphene which can be understood within one-electron picture, it is well recognised now that the electron-electron interaction can play an important role in understanding many electronic and transport properties of graphene [2, 3]. The aim of this work is therefore to develop an 
efficient numerical technique capable of treating the electron-electron interaction in graphene nanoribbons. To this end, we will develop a numerical solver for the Poisson's equation based on the finte difference and finite element methods (FDM and FEM), and combine them with the Thomas-Ferm approach for a system including a nanoribbon and a gate. By doing this, we test the Poisson solver and compare the results with the results of the quantum mechanical model of the same system based on the full tight-binding Hamiltonian with a Hatree potential [4], which will be introduced in the next section.

The development of the numerical PoissonThomas-Fermi solver is the first step toward a detailed quantum mechanical description of more complex quantum systems, for example graphene nanoribbons with edge disorder where the Anderson localisation can occur [5]. Our subsequent works will be aimed at the integrating of the efficient Poisson solver developed in the present paper with the full quantum-mechanical description of graphene nanoribbons.

\section{System under study}

We consider a graphene nanoribbon of the width $\omega$ situated on an insulating substrate with the relative permittivity $\varepsilon_{r^{\prime}}$. The dielectric is placed on a metallic bottom gate separated by the distance $d$. This is the simpliest standard graphene-gate set-up used in experiments. For simplicity we suppose that the graphene nanoribbon is surrounded by the rectangular insulating substrate, see Fig. 2.

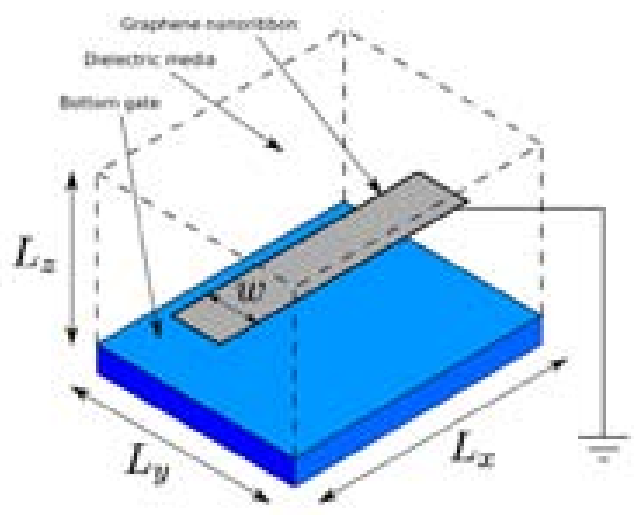

Fig. 2. Graphene nanoribbon - bottom gate system. The region is rectangle with the bottom gate in the botton boundary. The graphene nanoribbon is grounded.
Electrons in the graphene nanoribbon are described by a Thomas-Fermi approximation, which provides a simplified way to solve the quantummechanical problem. The dispersion relation of electrons in a graphene near the Dirac's cones is approximately linear, $E= \pm \hbar v_{\mathrm{F}} k_{\mathrm{F}}$ and the ThomasFermi equation reads

$$
\operatorname{sgn}(n(\mathbf{r})) \hbar v_{\mathrm{F}} \sqrt{\pi n(\mathbf{r})}-e V(\mathbf{r})=\mu,
$$

where $v_{\mathrm{F}}$ is the Fermi velocity, which for graphene is expressed in the carbon-carbon distance $a=0.142 \mathrm{~nm}$ and the hopping integral $t=2.77 \mathrm{eV}$ (see [2]), $\quad v_{\mathrm{F}}=\frac{3}{2} \frac{t a}{\hbar}$. We set the chemical potential to zero, $\mu=0$, which corresponds to the case when the graphene nanoribbon is grounded. The Coloumb's interaction of electrons is considered at Hartree level and the full tight-binding Hamiltonian for this case reads

$$
H=\sum_{\mathbf{r}} V_{\mathrm{H}}(\mathbf{r}) a_{\mathbf{r}}^{\dagger} a_{\mathbf{r}}-t \sum_{\mathbf{r}, \Delta} a_{\mathbf{r}}^{\dagger} a_{\mathbf{r}+\Delta},
$$

where $V_{\mathrm{H}}(\mathbf{r})$ is the Hartree potential created by graphene electrons and mirror charges. The manybody problem for this Hamiltonian is already solved [4] and our aim of the work is to solve the problem using the Thomas-Fermi equation (1) an compare it with the tight-binding approximation with the Hamiltonian (2).

The self-consistent potential $V(\mathbf{r})$ is obtained from the solution of the Poisson's equation

$$
\nabla\left(\varepsilon_{\mathrm{r}}(\mathbf{r}) \nabla V(\mathbf{r})\right)=-\frac{\rho(\mathbf{r})}{\varepsilon_{0}}=\frac{e n_{3 \mathrm{D}}(\mathbf{r})}{\varepsilon_{0}}
$$

Here $\varepsilon(\mathbf{r})$ is the relative permittivity, and $\rho(\mathbf{r})$ is the charge density in the graphene nanoribbon, which is expressed in the volume electron density $n_{3 \mathrm{D}}(\mathbf{r})$ $\rho(\mathbf{r})=-e n_{3 \mathrm{D}}(\mathbf{r})(e$ is the absolute value of an elementary charge). We write the subscript 3D to indicate that this density is three-dimensional. Later we will define a surface electron density without subscript. Note that in our graphene-bottom gate system (2) the relative permittivity is constant in the whole rectangle region $\varepsilon(\mathbf{r})=\varepsilon_{r^{\prime}}$. An effect of the bottom gate is included as the Dirichlet boundary condition in the bottom boundary of the rectangle region, $\left.V(\mathbf{r})\right|_{\left.\right|_{1}}=V_{b}$. In all other boundaries gion, $\left.V(\mathbf{r})\right|_{\text {at the bottom gate }}=V_{\mathrm{bg}}$. In all other boundaries
we use homogeneous Neumann boundary conditions, $\partial V(\mathbf{r}) / \partial \mathbf{n}=0$, which indicate that the system tions, $\partial V(\mathbf{r}) / \partial \mathbf{n}=0$, which indicate that the system
is electrically neutral and there are no charges outside system's boundaries.

In the next section we provide a more detailed description of the methods to numerically solve the Poisson's equation and in the section 3 we describe the self-consistent method which we use to simultaneously solve both Poisson's and Thomas-Fermi equations.

\section{Numerical methods for the solution of} Poisson's equation

In order to solve the Poisson's equation (3) numerically we use the finite difference and the finite element discretisation. In this section we will describe both discretisation methods.

The finite difference method is one of the most easily implemented discretisation methods. One has to introduce a three-dimensional structured grid, a set of points that the whole rectangular region is divided into a finite num ber of smaller cubes. Suppose that $N_{x}, N_{y}$ and $N$ are the numbers of cubes in each direction and that $h$ is the size of the cube's edge. Then the size of the region is $L_{x}=h \cdot N_{x^{\prime}} L_{y}=h \cdot N_{j} L_{z}=h \cdot N_{z}$ The electrostati The electrostatic potential and the volume elecron density in each grid point is expressed as $V_{i, j, k}=V(i h, j h, k h), n_{i, j, k}^{3 \mathrm{D}}=\mathrm{n}_{3 \mathrm{D}}(i h, j h, k h)$. The Poisson's equation is discretised by approximat ing the second derivatives $\partial^{2} V / \partial q^{2}, q \equiv x, y, z$ (or additionally the first derivatives $\partial V / \partial q$ if required) in the interior grid points (in the grid points which are not in the boundary) using the central difference formulas:

$$
\left.\frac{\partial^{2} V}{\partial x^{2}}\right|_{i, j, k} \simeq \frac{V_{i+1, j, k}+V_{i-1, j, k}-2 V_{i, j, k}}{h^{2}}
$$

and similar expressions for the other second derivatives. For all interior grid points $(i, j, k)$ we get a system of linear equations:

$$
V_{i+1, i, j}+V_{i-1, j, k}+V_{i, j+1, k}+V_{i, j-1, k}+
$$

$$
+V_{i, j, k+1}+V_{i, j, k-1}-6 V_{i, j, k}=h^{2} \cdot \frac{n_{i, j, k}^{3 \mathrm{D}}}{\varepsilon_{\mathrm{r}} \varepsilon_{0}}
$$

where the electrostatic potential values $V_{i, j}$ are unknowns and the right- hand side is proportional to the volume electron density values $n_{i, j, j}^{3 \mathrm{D}}$.

The boundary conditions are not imposed yet and the system of linear equations does not have a unique solution. The Dirichlet boundary condition in the bottom gate is included by setting $V_{\text {ipo }}=V$ Similarly the Neumann boundary conditions on all other boun auxiliary conditions $V_{0, j, k}=V_{1, j, k^{k}}, V_{N_{\mathrm{x}}+1, j, k}=V_{N_{\mathrm{x}, j, k}}$ $V_{i, 0, k}=V_{i, 1, k}, V_{i, N_{\mathrm{y}}+1, k}=V_{i, N_{\mathrm{y}}, k}$ and $V_{i, j, \mathrm{~N}_{\mathrm{z}}+1}=V_{i, j, N_{\mathrm{z}}}$ which are derived from the approximation of the first derivatives of the potential $V$ in these boundaries, for example,

$$
\left.\frac{\partial V}{\partial x}\right|_{0, j, k} \simeq \frac{V_{1, j, k}-V_{0, j, k}}{h}=0
$$

gives us the first auxiliary condition. By imposing all boundary conditions we relate the potential values in the boundaries with the values in the interio grid points and get a smaller number of unknowns

Although the finite difference method of discretising the Poisson's equation is simple, it has sevIt also fails when we want to consider some part of the domain with more precision than other, fo example in our case we want to calculate the potential in the graphene strip with more precision than the potential in the boundaries. In these cases one usually uses another popular method, the finite element method. The basic idea of this method is to divide the whole domain into a finite number of sub-domains (called elements) and then approximate electrostatic potential in each element with some interpolation function, which is characterised by some finite number of parameters. Finally, one constructs a system of linear equations, where these parameters are unknowns, solves it and use the solution to calculate the potential in an arbitrary domain point or other quantities, for example the gradient field of the potential. 
In our calculations we discretise the Poisson's bitrary point of the whole rectangular domain is equation (Eq. 3) using a standard finite-element given as a sum of the potential in all tetrahedron Galerkin formulation [6]. We divide the whole elements, rectangular domain into the simplest linear tetrahedron elements and construct a system of linear equations for each element $e$ :

$$
\mathbf{A}^{(e)} \mathbf{V}^{(e)}=\mathbf{b}^{(e)} .
$$

$$
V(\mathbf{r})=\sum V^{(e)}(\mathbf{r}),
$$

and the global system of linear equations

$$
\mathbf{A V}=\mathbf{b}
$$

Here $\mathbf{A}^{(e)}$ is an elemental stiffness matrix, $\mathbf{V}^{(e)}$ is a vector of the values of the electrostatic potential in the vertices of $e$-th tetrahedron, and $\mathbf{b}^{(e)}$ is a right-hand side vector of the linear system. The electrostatic potential in an arbitrary point in the element is expressed in $\mathbf{V}^{(e)}$ and shape functions $\mathbf{u}^{(e)}=\left(u_{1}^{(e)}(\mathbf{r}), \ldots, u_{4}^{(e)}(\mathbf{r})\right)$

$$
V^{(e)}(\mathbf{r})=\mathbf{u} \cdot \mathbf{V}^{(e)} \text {. }
$$

is constructed by assembling all elemental system (Eq. 7) and changing into the global numbering of nodes: $\mathbf{V}=\left(V_{1}, \ldots, V_{N}\right)$, where $N$ is the total number of nodes (points where the vertices of tetrahedrons resides). The system of linear equation (Eq. 12) with assembled systems, whose matrix and r. h. s. vector entries are given by (Eq. 10)

(8) automatically includes homogeneous Neumann boundary conditions. In the final part of the The shape functions $u_{m}^{(e)}(\mathbf{r})$ of the linear tet- system construction we impose the Dirichlet rahedron are the fractions of the volumes $\Delta_{P 234}$, boundary conditions using a boundary penalty $\Delta_{1 P 34}, \Delta_{12 P 4}, \Delta_{123 P}$ and $\Delta_{1234}$ of the tetrahedrons with method [7].

the vertices $\{P, 2,3,4\},\{1, P, 3,4\},\{1,2, P, 4\}, \quad$ The Poisson's equation (3) requires the volume $\{1,2,3, P\}$ and $\{1,2,3,4\}$, respectively, where 1 , electron density $n(\mathbf{r})$, but the solution of the 2,3 and 4 are the vertices of the whole element Thomas-Fermi equation is expressed in the surface tetrahedron and $P$ is an arbitrary point $\mathbf{r} \equiv(x, y, z) \quad$ electron density $n(\mathbf{r})$. In order to numerically solve inside the tetrahedron:

$$
\begin{aligned}
& u_{1}^{(\mathrm{e})}(\mathbf{r})=\Delta_{P 234} / \Delta_{1234^{\prime}} \\
& u_{2}^{(\mathrm{e})}(\mathbf{r})=\Delta_{1 P 34} / \Delta_{1234^{\prime}} \\
& u_{3}^{(\mathrm{e})}(\mathbf{r})=\Delta_{12 P 4} / \Delta_{1234^{\prime}} \\
& u_{4}^{(\mathrm{e})}(\mathbf{r})=\Delta_{123 \mathrm{P}^{2}} / \Delta_{1234^{\circ}}
\end{aligned}
$$

If the point $P$ is outside the element, the shape function of that element is equal to zero.

In the standard Galerkin finite-element formulation entries of the stiffness matrix $\mathbf{A}^{(e)}$ and r. h. s. vector $\mathbf{b}^{(e)}$ are

$$
\begin{aligned}
& A_{m, n}^{(e)}=\int_{\Omega^{(e)}} u_{m}^{(e)}(\mathbf{r}) u_{n}^{(e)}(\mathbf{r}) \mathrm{d} \mathbf{r}, \\
& b_{m}^{(e)}=-\int_{\Omega^{(e)}} \frac{e n_{3 \mathrm{D}} \mathbf{r}}{\varepsilon_{\mathrm{r}} \varepsilon_{0}} u_{m}^{(e)}(\mathbf{r}) \mathrm{d} \mathbf{r},
\end{aligned}
$$

where we integrate in the whole tetrahedron volume $\Omega^{(e)}$. Finally, electrostatic potential in an ar- both equations, we need to relate these densities. In the finite difference approximation the relation between the surface and volume electron densities in the graphene strip is

$$
n_{3 \mathrm{D}}(\mathbf{r})=n(\mathbf{r}) / h,
$$

where $h$ is the distance between two nearest grid points of the finite difference approximation. In the finite element approximation this relation has the same form, but we define the distance $h$ as a maximum height of the elements (maximum space in the $z$ direction occupied by the element) which contain graphene strip points. In the section 5 we will show that we generate a structured mesh near the strip, so all tetrahedron elements which contain strip points have the same height. We will call this height a strip thickness.

Both the finite difference and the finite element discretisation methods give us a sparse global stiffness matrix $\mathbf{A}$. For the actual calculation we use the LibMesh $\mathrm{C}++$ finite element library [8]. For an automatic mesh generation we use the GMSH software $[9]$

\section{Self-consistent calculation}

The numerical solution $(n(\mathbf{r}), V(\mathbf{r}))$ to both Poisson's and Thomas-Fermi equations is calculated using a self-consistent method, which is graphically represented in Fig. 3.

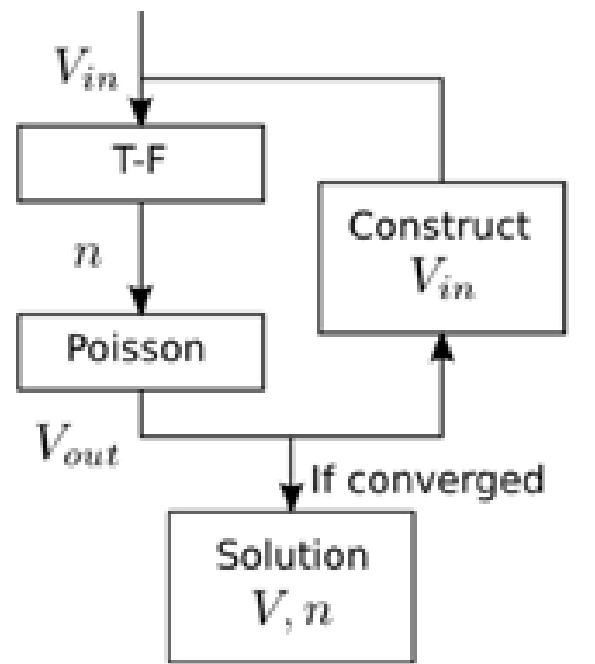

Fig. 3. The schematic representation of the self-consistent method.

We start with some initial guess for the potential $V_{\text {in }}(\mathbf{r})$ and use it to calculate the electron density $n(\mathbf{r})$ from the Thomas-Fermi equation (1). Then we use this electron density to calculate the new the discretised Poisson's equation $(5,12)$ the electrostatic potential is represented as a finite dimensional vector $\mathbf{V}$. Having the input and output vec tors $\mathbf{V}_{\text {in }}$ and $\mathbf{V}_{\text {out }}$ we calculate the relative difference between them, which we define as

\section{$r=\frac{\left\|\mathbf{V}_{\text {out }}-\mathbf{V}_{\text {in }}\right\|}{\left\|\mathbf{V}_{\text {in }} \mid+\right\| \mathbf{V}_{\text {out }} \|}$}

where $\|\cdot\|$ is an Euclidean $\left(l^{2}\right.$-norm) norm. If this norm is not small enough (the input and output vectors differ too much), we construct the new potentia mi and Poisson's equations again. In every iteration we check the convergence, the relative difference between the input and output potentials, and stop if it becomes very small. In our calculations we choose the convergence criterion $r<10^{-5}$.
In each iteration the input potential $\mathbf{V}_{\text {in }}$ can be constructed in various ways. The simplest construction reads

$$
\mathbf{V}_{\text {in }}^{k+1}=\delta \mathbf{V}_{\text {out }}^{k}+(1-\delta) \mathbf{V}_{\text {in }}^{k}
$$

where $k$ is an iteration number and $\delta$ is some smal real positive parameter. Using this construction a single self-consistent calculation can take about 500-2000 iterations. Note that we are currently implementing the Broyden's second method [10] which can decrease the number of iterations significantly.

\section{Main results}

A typical generated mesh used in our calculation is shown in Fig. 4. The mesh is fine and structured near the ribbon and rather coarse-grained in othe parts of the computational domain. The purpose of the fine-structured mesh near the graphene nanoribbon is to model the strip, which has the same thickness in all its points.

The main results of self-consistent calculations, distribution of electron density and electrostatic potential in the strip, are presented in Figs. 5 and

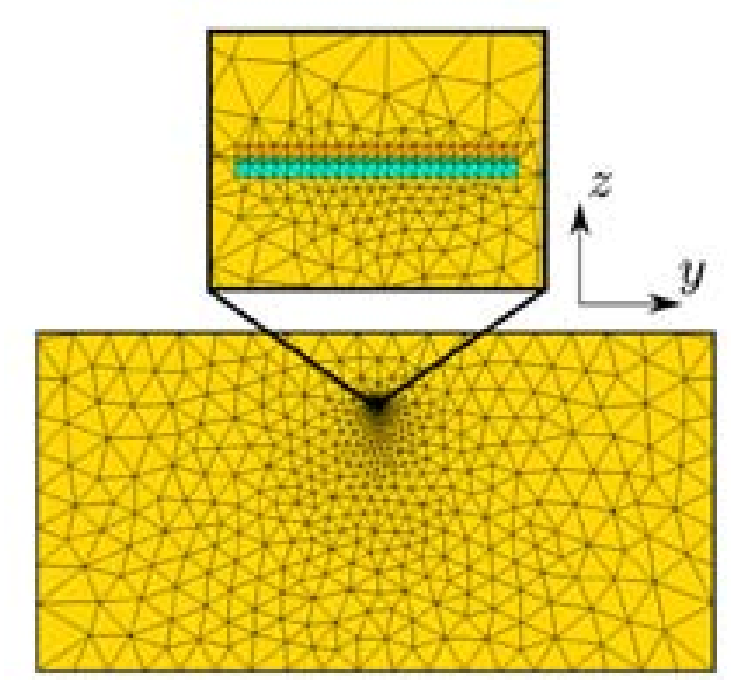

Fig. 4. Generated mesh (only $y z$ plane is presented). I the upper figure we show how mesh is generated near the graphene nanoribbon, which is extended in the direction 
6 . The distributions are averaged in the $x$ direction because of a translational symmetry. The ThomasFermi model is compared with a corresponding quantum-mechanical tight-binding model, which is described in Ref. [4]. The size of the computational domain is $\left\{L_{x} L_{\nu}, L_{z}\right\}=\{100 \mathrm{~nm}, 1000 \mathrm{~nm}$ $500 \mathrm{~nm}\}$, the strip width is $20 \mathrm{~nm}$, the distance from the bottom gate to the strip is $d=100 \mathrm{~nm}$, the elec trostatic potential in the bottom gate is $V_{b}=5 \mathrm{~V}$ and the relative permittivity of the dielectric media is $\varepsilon=3.9$. We express the electrostatic potential in energy $t$ units (for an electron $1 \mathrm{~V}$ corresponds to $1 \mathrm{eV})$. In both figures the red line corresponds to the finite element Poisson-Thomas-Fermi model the light blue line with points corresponds to the finite difference Poisson-Thomas-Fermi model and the blue points correspond to the tight-binding Hartree model.

The results show a good correspondence with the tight-binding model. Because of the electrostatic repulsion of electrons, the electron density near the edges is larger than in the nanoribbon center. Notice that in the model based on the tightbinding approximation (blue points) electron density varies significantly near the edges of the graphene nanoribbon. This is because the model takes into account all graphene lattice points and electron density can differ in two graphene sublattices significantly (in $A$ and $B$ sub-lattices, see Fig. 1). On the other hand, the Thomas-Ferm equation is not a very good approximation near the edges of the physical system when elector density varies rapidly.

By comparing the results of the finite elemen and finite difference methods, we see that FEM better corresponds to the tight-binding method. FDM considers only several grid points in the width of the graphene nanoribbon and approximation is not as good as in FEM.

The convergence of the self-consistent algorithm of Poisson's and Thomas-Fermi equation mainly depends on the parameter $\delta$ (Eq. 15) an on the electrostatic potential of the bottom gate $V_{\mathrm{bg}}$ Usually the number of self-consistent iterations decreases by increasing the parameter $\delta$ until some critical value $\delta$, after which the iterations start to diverge (when $\delta>\delta_{c}$ )

The results of one convergence test are presented in Fig. 7. In each calculation we used differen values of the parameter $\delta$. The size of the region,

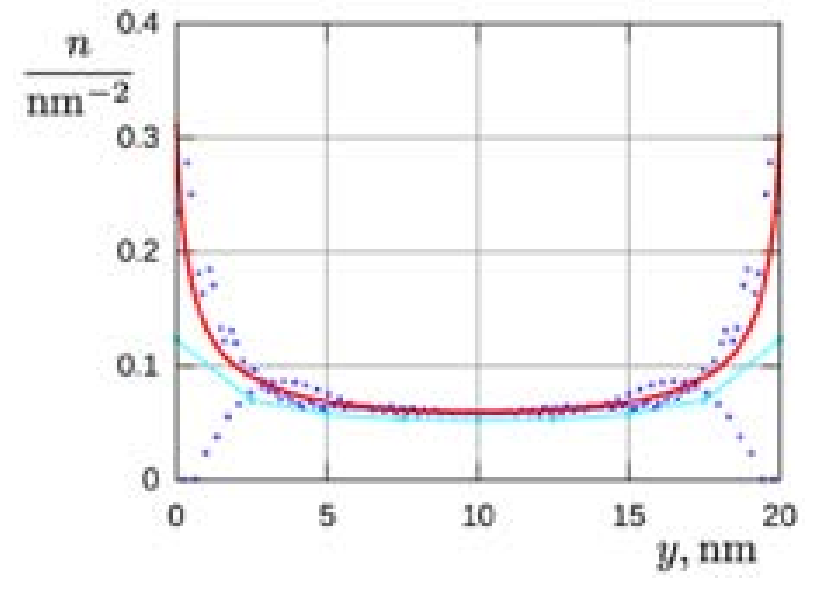

Fig. 5. Distribution of surface electron density in the graphene nanoribbon width.

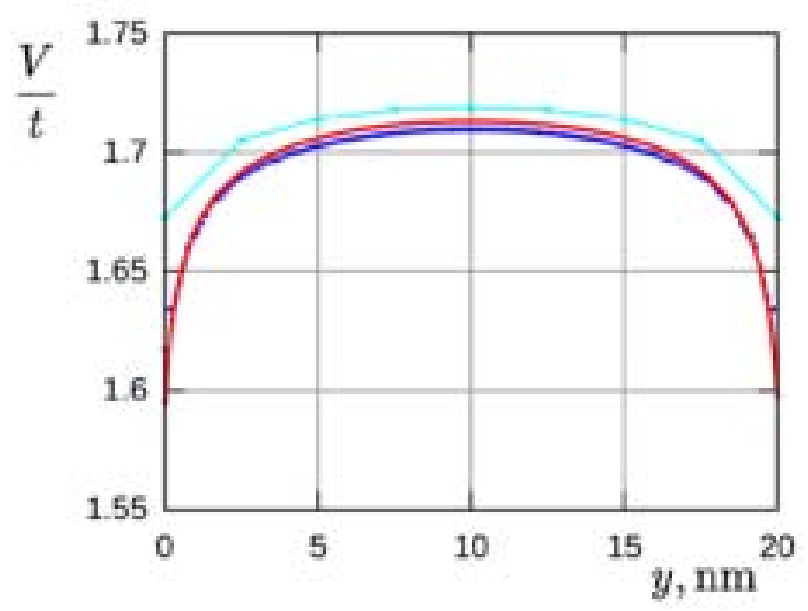

Fig. 6. Dependence of the electron energy difference between the graphene nanoribbon and the bottom gate on the position in the strip width. The energy difference is expressed in $t=2.77 \mathrm{eV}$ units.

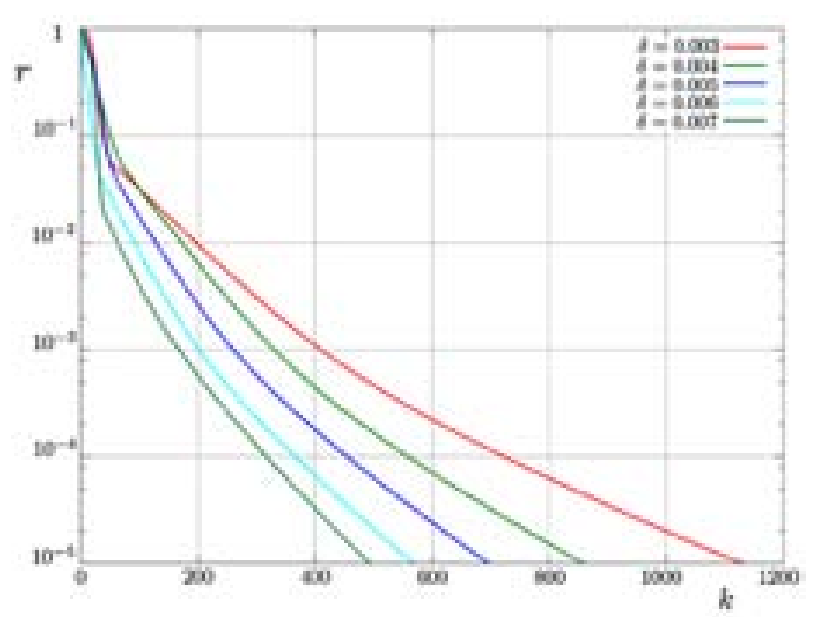

Fig. 7. Convergence of the self-consistent algorithm of finding a solution to the Poisson's (3) and Thomas-Ferm equations (1). $k$ is the number of iterations, $r$ is the relative error (14) and $\delta$ is a small real positive parameter (15).

strip width, distance from the bottom gate to the Acknowledgments strip and relative permittivity have the same values as in the previously presented results. complex by the Rearch Council of Lithuania MIP-123. quires less computer memory and converges faster. The reason of this is that in FEM we need to consider a smaller number of potential values in the computation domain than in FDM.

\section{Conclusions}

We developed the numerical solver of the Poisson's equation (3) and tested it for a system consisting of a gate and graphene nanoribbon, in which electron are described in the Thomas-Fermi approximation. The results of the self-consistent Poisson-Thomas(Termi correspondence with the results of the quantum-mechanical tightbinding calculations [4].

The quantum-mechanical tight-binding calculations give more precise results than the semi-classical Thomas-Fermi equation, which may not include such edge effects as Anderson localisation. However the self-consitent tight-binding calculation treats the bottom gate as a collection of mirror charges of electrons and it cannot be used for more complex systems. The results of this work show that the Poisson's equation can be successfully used to calculat the self-consistent potential without mirror charges.

\section{References}

[1] K.S. Novoselov A.K. Geim, S.V. Morozov, D. Jiang Y. Zhang S.V. Dubonos, IV. Grigorieva, and A.A. Firsov, Science 306, 666 (2004)

[2] A.H. Castro Neto F. Guinea, N.M.R. Peres, K.S. Novoselov, and A.K. Geim, Rev. Mod. Phys. 81, 109 (2009).

3] S. Das Sarma, S. Adam, E.H. Hwang, and E. Rossi, Rev. Mod. Phys. 83, 407 (2011).

A.A. Shylau, J.W.K los, and I.V. Zozoulenko, Phys, Rev. B 80, 205402 (2009).

[5. M. Evaldsson, I.V. Zozoulenko, H. Xu, and T. Heinzel, Phys. Rev. B 78, 161407 (2008).

6]. J.L. Volakis, A. Chatterjee, and L.C. Kempel, Finite Element Method for Electromagnetics, Antennas, Microwave Circuits and Scattering Applications (IEEE Press, New York, 1998).

7]. B.W. Clark and D.C. Anderson, Finite Elem. Anal. Design 39, 387 (2003).

[8]. B.S. Kirk, J.W. Peterson, R.H. Stogner, an G.F. Carey, Eng. Comput. 22, 237 (2006)

[9]. C. Geuzaine and J.-F. Remacle, Int. J. Numer. Meth. Eng. 79, 1309 (2009)

0]D. Singh, H. Krakauer, and C.S. Wang, Phys. Rey. B 34, 8391 (1986)

\section{GRAFENO JUOSTELĖS ELEKTROSTATINĖS UŽTŪROS MODELIAVIMAS TOMO IR FERMIO BEI PUASONO LYGTIMIS}

T. Andrijauskas ${ }^{\mathrm{a}}$, A. A. Shylau ${ }^{\text {b }}$ I. V. Zozoulenko ${ }^{\mathrm{b}}$

a Vilniaus universiteto Teorineis fizikos ir astronomijos institutas, Vilnius, Lietuva

${ }^{\mathrm{b}}$ Linčiopingo universiteto Mokslo ir technologiju katedra (MTK), Švedija

Aprašomos paprastos grafeno juostelès ir lauko kontakto sistemos, pateikti skaitmeniniai metodai Puasono bei Tomo ir Fermio lygtims spresti, kuriais modeliuojamas elektronu judejjimas. Puasono lygtis sprendžiama naudojant baigtiniu skirtumu ir baigtiniu elementu metodus. Naudojant Tomo ir Fermio bei Puasono lygtis suskaičiuojama elektrostatinis potencialas ir paviršinis elektronụ tankis grafeno juostelëje. Galiausiai nagrinëjamas modelis lyginamas su Hartrio modeliu. Pagrindinia rezultatai rodo gerą modelių sutapimą. Aprašytas elek(rostatinio potencialo skaiciavimo büdas taikant Puasono lygtị gali būti naudojamas sudètingesnèse sistemose. 